\title{
Wavelength-band Transformation Method of Gray-body Infrared Image Using Surface Temperature Estimation
}

\author{
Bong-Seob Kim ${ }^{1}$, Hyuk-Ju Kwon ${ }^{1}$, Tae-Wuk Bae ${ }^{1}$, Sang-Ho Ahn ${ }^{2}$, \\ Young-Choon $\mathrm{Kim}^{3}$ and Kyu-Ik Sohng ${ }^{1}$ \\ ${ }^{1}$ School of Electronics Engineering, Kyungpook National University, \\ ${ }^{2}$ Dept. of Electronic Engineering, Inje University, \\ ${ }^{3}$ Dept. of Information and Communication Security, Youngdong University \\ bskim@ee.knu.ac.kr,olin1223@ee.knu.ac.kr,nanninggo@gmail.com, \\ elecash@inje.ac.kr,yckim@yd.ac.kr,and kisohng@ee.knu.ac.kr
}

\begin{abstract}
Infrared (IR) imaging systems obtain thermal images by converting surface temperature into two-dimensional images by measuring $I R$ radiation from an object's surface. This research proposes a wavelength-band transformation method to convert an IR image of an arbitrary wavelength-bandwidth into an IR image of another wavelength-bandwidth. We estimate the temperature of an arbitrary wavelength by obtaining the radiance from the black body radiation theory and the linear transformation function of the gray level from the radiance. We generate an IR image for another wavelength-bandwidth by calculating the radiance of another wavelength-bandwidth using the estimated temperature. The validity of the proposed method was verified through simulations. The IR target image was generated using RadThermIR software, and images, acquired from a thermal observation device, were used as IR background image.
\end{abstract}

Keywords: infrared image, wavelength-band translation, object surface temperature estimation, transformation function

\section{Introduction}

Infrared (IR) images are broadly used for military, civilian, and industrial purposes because it can present the IR wavelength-bandwidth that cannot be seen by humans. And IR simulators are commonly used for development and performance evaluation of systems using IR. The necessity of IR simulators is gradually increasing in terms of IR system development for its accompanying safety reasons and cost reduction, as well as concept analysis, theory formulation, and performance evaluation of the system. In such IR simulators, target and background should be adequately modeled as IR signatures of brightness depending on temperature. There has been a lot of research on IR signaturemodeling methods with relevant theoretical background such as radiation theory, heat transfer theory, and atmospheric transfer characteristics [1-8]. IR images mainly use a shortwave IR (SWIR) bandwidth, middle wave IR (MWIR) bandwidth, and long wave IR (LWIR) bandwidth by atmospheric transfer characteristics [9-13]. In order to acquire an IR image of three wavelength-bandwidths, a thermal observation device is required for each wavelength-bandwidth, and there is difficulty in simultaneously acquiring an identical target. 
Ahn et al., proposed a bandwidth conversion method which convert an IR image of single bandwidth into other two bandwidth IR images [14]. In this method, assuming all objects included in an IR image are gray bodies of identical emissivity, the bandwidth conversion method was derived using maximum and minimum temperature information corresponding to maximum and minimum values of the gray level. However, this method is very complicated, and has a limitation in that all the objects should be gray bodies that have the same emissivity.

In order to solve the mentioned difficulty, this study proposes a wavelength-band transformation method to convert a wavelength-bandwidth by estimating the surface temperate of an object. The study uses the premise that radiance, which is obtained by each wavelength-bandwidth depending on temperature from the black body radiance theory, is proportional to gray level of an IR image. The transfer function of radiance to gray level is obtained by using a mapping function of a certain two-pixel gray level and its corresponding temperature. The temperature of an object can be estimated from the gray level on all pixels of an IR image by inverting this transfer function. IR images of other wavelength-bandwidths are generated by converting them to gray level after calculating radiance based on the estimated temperature. The proposed method, enabling the conversion of wavelengthbandwidth depending on emissivity and temperature, is possible if the object's emissivity is known.

The validity of the proposed method was verified through MATLAB simulation. The IR target image is generated using RadThermIR software, and images, acquired from a thermal observation device, are used as the IR background images.

\section{Radiance Theory}

A black body means an ideal IR radiance body. The spectral radiance of a black body as to a certain wavelength $\lambda$ and temperature $T$ is given by

$$
\mathrm{L}(\lambda, T)=\frac{C_{1}}{\lambda^{5}\left(\exp \left(\frac{C_{2}}{\lambda T}\right)-1\right)}\left[W /\left(\mathrm{cm}^{2} \mu m s r\right)\right]
$$

through Planck's Law of Radiation, where the radiation constants $C_{1}$ and $C_{2}$ are

$$
\begin{aligned}
& C_{1}=1.191 \times 10^{4}\left[\mathrm{Wum}^{4} /\left(\mathrm{cm}^{4} \mathrm{sr}\right)\right] \\
& C_{2}=1.428 \times 10^{4}[\mu \mathrm{mK}]
\end{aligned}
$$

where $\varepsilon(\lambda)$ is the spectral emissivity of an object and an object represents a black body if $\varepsilon(\lambda)=1$ and a gray body if $\varepsilon(\lambda)<1 . \lambda_{1}$ and $\lambda_{2}$ are wavelengths. The radiance of an object is given by

$$
\mathrm{L}(T)=\int_{\lambda_{1}}^{\lambda_{2}} \frac{C_{1}}{\lambda^{5}\left(\exp \left(\frac{C_{2}}{\lambda T}\right)-1\right)} d \lambda\left[W /\left(\mathrm{cm}^{2} s r\right)\right]
$$

Figure 1 illustrates the atmospheric transfer characteristics of a radiant energy at IR wavelength-bandwidth. Radiant energy cannot be transferred at certain wavelengthbandwidths as it is absorbed by oxygen, carbon dioxide, ozone and vapor in the air. 


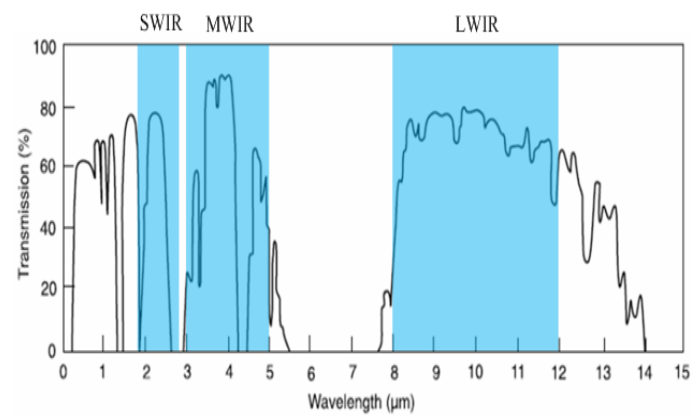

Figure 1. Atmosphere transmission characteristics of IR band

According to the atmospheric permeation characteristics, the bandwidth $\lambda_{1} \sim \lambda_{2}$ ranges $1.9 \sim 2.9 \mu \mathrm{m}$ at SWIR, $3 \sim 5 \mu \mathrm{m}$ at MWIR and $8 \sim 12 \mu \mathrm{m}$ at LWIR in general IR system.

Figure 2 illustrates the spectral radiance depending on the temperature of the black body, obtained by Plank's Law of radiation in Equation (1). The result of the radiance depending on the temperature by Equation (3) as three bandwidths is seen in Figure 3.

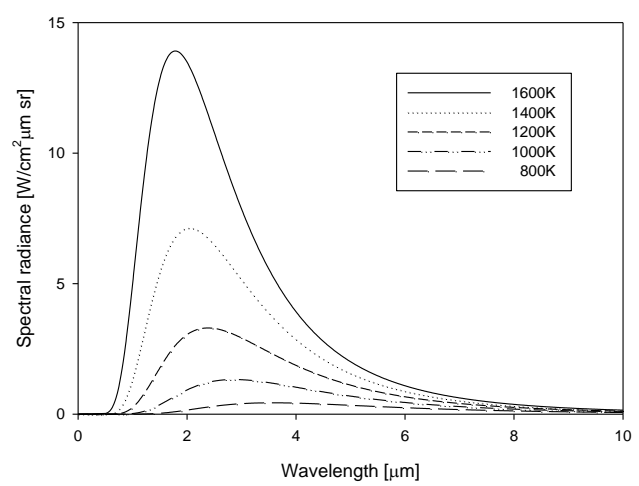

Figure 2. Spectral radiance of blackbody

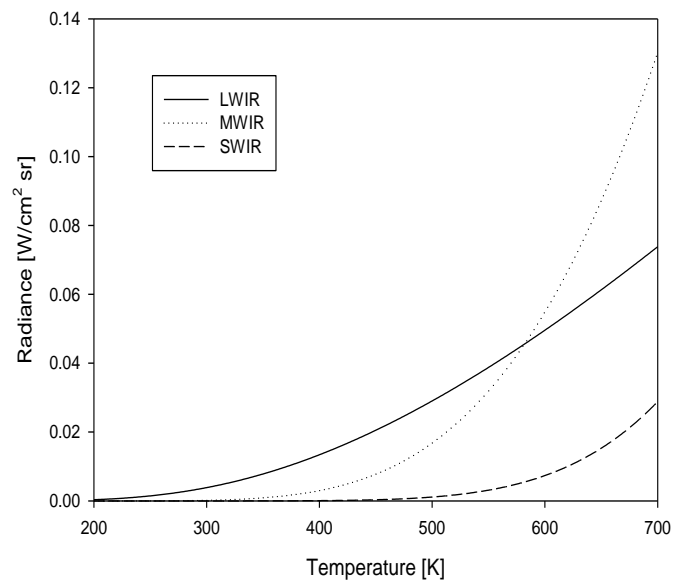

Figure 3. Radiance of wavelength-band for temperature 


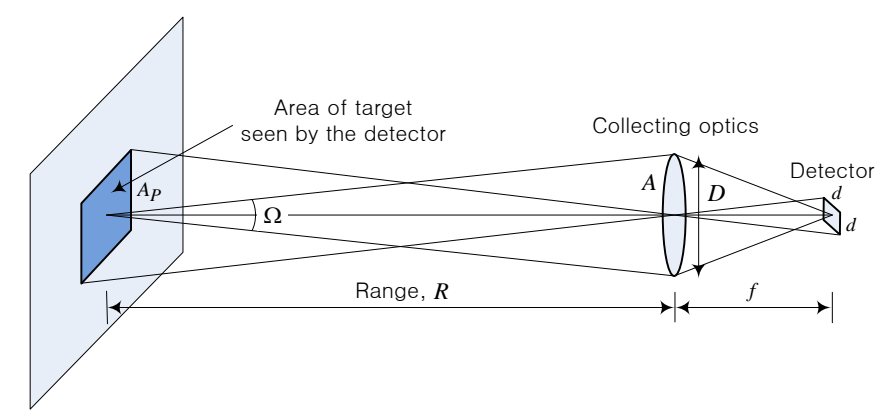

\section{Figure 4. Geometric configuration of target and detector}

Figure 4 illustrates the geometric configuration of a target and a detector. The target is located at $R$ distance from the IR sensor. In case $L$ is the lens diameter of the sensor, $A$, $d \times d$, and $f$ represent the lens area, detector size, and focus distance respectively. And the target area, shown from the detector, is given by

$$
A_{p}=\left(\frac{d R}{f}\right)^{2}[\mathrm{~cm}]
$$

and the solid angle of the detector, seen from the target, is as follows;

$$
\Omega=\frac{A}{R^{2}}=\frac{\pi D^{2}}{4 R^{2}}[s r]
$$

The output voltage of the detector is obtained as the following;

$$
\begin{aligned}
V_{\text {det }} & =A_{p} \Omega L(T) \int_{\lambda_{1}}^{\lambda_{2}} \tau_{a m b}(\lambda) \tau_{\text {opt }}(\lambda) S(\lambda) d \lambda \\
& =\frac{\pi}{4}\left(\frac{d D}{f}\right)^{2} L(T) \int_{\lambda_{1}}^{\lambda_{2}} \tau_{\text {amb }}(\lambda) \tau_{\text {opt }}(\lambda) S(\lambda) d \lambda[V]
\end{aligned}
$$

where $\tau_{\text {amb }}(\lambda)$ and $\tau_{\text {opt }}(\lambda)$ represent the spectral transmissivity of atmosphere and optics and $S(\lambda)$ is the spectral responsivity $[V / W]$. If $\tau_{\text {amb }}(\lambda), \tau_{\text {opt }}(\lambda)$, and $S(\lambda)$ are time invariant systems, the integrals of their wavelength-bandwidths have constant values and $A_{p}, \Omega$ are also constants. This implies that the output of the detector is directly proportional to the radiance $L(T)$ depending on the temperature. Although $L(T)$ is a function by spectral emissivity $\varepsilon(\lambda)$ as in Equation (4), $L(T)$ becomes a function by only temperature $T$. And it becomes a constant $\varepsilon$ in case the object is a black body or a gray body.

If the detector is a two-dimensional image detector, the gray level of an IR image is also proportional to the output voltage of the detector. Therefore, it can be said that the radiance $L(T)$ of a certain wavelength-bandwidth regarding the object temperature $T$ is proportional to gray levels of an IR image. 


\section{Proposed Wavelength-bandwidth Conversion Method}

In this paper, a wavelength-band conversion method, converting into an IR image of another wavelength-bandwidth from an IR image of an arbitrary bandwidth, has been proposed. The conversion relationship for the object temperature to the radiance and the gray level to the radiance is shown in Figure 5.

In case the object is a gray body, the size is inverse proportional to the emissivity, on the other hand, the radiance curve is identical relative to that of the black body $(\varepsilon=1.0)$ because the emissivity $\varepsilon$ is a constant. Figure 5 is an example of an object with a pixel of maximum gray level $G_{\max }$ and maximum temperature $T_{\max }$ for emissivity 0.8 and an object with the pixel of minimum gray level $G_{\min }$ and minimum temperature $T_{\min }$ for emissivity 0.6.

$$
\begin{aligned}
& L\left(T_{\max }\right)=\int_{\lambda_{1}}^{\lambda_{2}} \frac{\varepsilon_{1}(\lambda) C_{1}}{\lambda^{5}\left(\exp \left(\frac{C_{2}}{\lambda T_{\max }}\right)-1\right)} d \lambda\left[W / \mathrm{cm}^{2} s r\right] \\
& L\left(T_{\min }\right)=\int_{\lambda_{1}}^{\lambda_{2}} \frac{\varepsilon_{2}(\lambda) C_{1}}{\lambda^{5}\left(\exp \left(\frac{C_{2}}{\lambda T_{\min }}\right)-1\right)} d \lambda\left[W / \mathrm{cm}^{2} s r\right]
\end{aligned}
$$

The radiance $L(T)$ of a certain wavelength-bandwidth $\left(\lambda_{1} \sim \lambda_{2}\right)$ depending on temperature can be obtained using Equation (3) through the Law of Black Body Radiation, and the conversion function of the gray level as to the radiance is a primary function because the gray level $G$ of an IR image is proportional to radiance. The gradient of the conversion function is

$$
a=\frac{G_{\max }-G_{\min }}{L\left(T_{\max }\right)-L\left(T_{\min }\right)}
$$

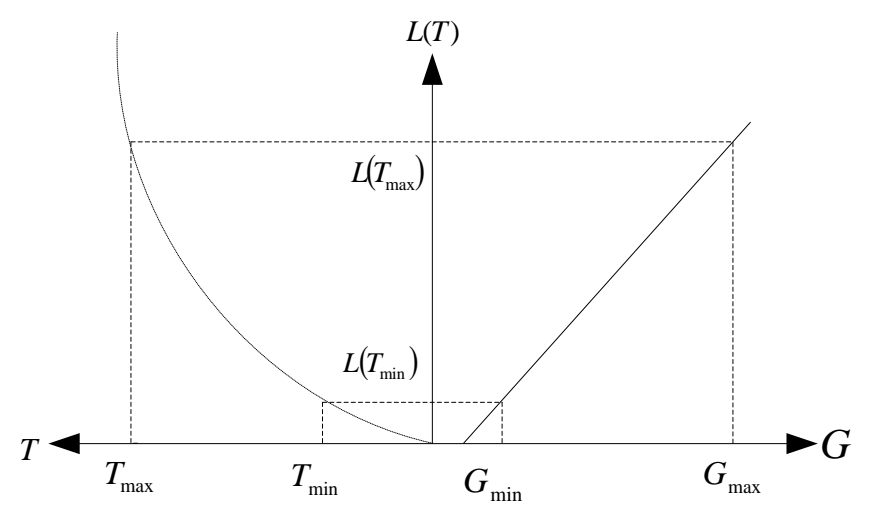

Figure 5. Illustration of radiance and gray-level according to temperature

And the conversion function can be expressed as

$$
G(L)=a\left[L-L\left(T_{\min }\right)\right]+G_{\min }
$$


It assumed that an IR image of a certain wavelength-bandwidth is given and the temperature and emissivity of an object corresponding to at gray-levels of least 2 pixels in the image are known. The bigger difference of the temperature difference and the gray level of the 2 pixels, the better it is. As in Figure 1, if we assume 2 pixels of an image have minimum and maximum temperature and gray levels, we can obtain $G_{\max }$ and $G_{\min }, T_{\max }$ and $T_{\min }$. The step for wavelength-bandwidth transformation is as follows.

Step 1) Calculate the radiance as to the maximum and minimum temperatures. If the temperature of an object corresponding to maximum gray level $G_{\max }$ on the IR image of a certain wavelength-bandwidth is $T_{\max }$, the radiance $L\left(T_{\max }\right)$ can be obtained by Equation (7). Also, $L\left(T_{\min }\right)$ can be obtained when the temperature on the minimum pixel of the object is $T_{\min }$ and the emissivity is $\varepsilon_{2}$.

Step 2) Calculate the conversion function of the radiance to gray level. Obtain the conversion function of the radiance to gray level $G(L)$ by Equation (7) and Equation (8).

Step 3) Estimate the temperature for all the pixels of IR images. The inversion formula for radiance $L$ from the gray level $G$ can be obtained from Equation (9), similarly to Equation (10).

$$
L(G)=\frac{G-G_{\min }}{a}+L\left(T_{\min }\right)
$$

Also, in order to estimate the temperature of the target surface, we use a look-up table for inverse model of the Equation (2). Look-up table can be used to calculate the temperature $T$ from the radiance $L$. The look-up table can be obtained by mapping the radiance $L$ from the gray level $G$ as shown in Figure 6.

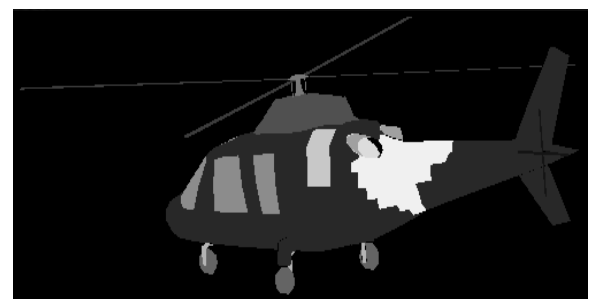

(a)

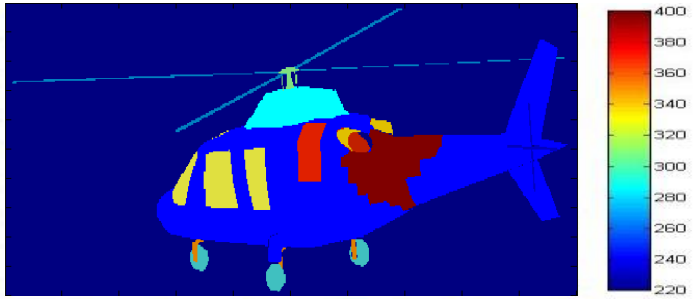

(b)

Figure 6. Estimate temperature converted wavelength-band image: (a) Modeled Agusta helicopter images and (b) estimated temperature image

Step 4) Calculate the radiance of other bandwidths using the temperature $T$ and the emissivity $\varepsilon$ on all the pixels. It can be calculated by using Equation (3) and the pre-defined wavelength-bandwidth $\lambda_{1} \sim \lambda_{2}$ can be varied into other bandwidth. 
Step 5) Generate an image of other wavelength-bandwidths using the gray level conversion formula $G(L)$ from the radiance obtained from all the pixels. The gray level conversion function can be used as Equation (1). Here, $0<g \leq 1$ is a constant to control the contrast, and $b$ is brightness control constant. If $g=1$ and $b=0$, the gray level is distributed from 0 to 255 .

$$
G(L)=\frac{255\left(L-L_{\min }\right)}{L_{\max }-L_{\min }}+b, 0<G(L) \leq 255
$$

\section{Simulation Result}

A simulation is performed in order to investigate the validity of proposed wavelengthband conversion method of an IR image. As a typical target image, Figure 7(a) shows a 3D CAD model of Agusta helicopter, composed of meshs. Figure 7(b) is a modeled IR target image of Agusta helicopter for LWIR, using RadthermIR developed by TAI corp. Figure 7(c) and 7(d) are the converted wavelength-band image for SWIR and MWIR based on Figure 7(b) with $T_{\min }=0, T_{\max }=600$ and $G_{\min }=0, G_{\max }=255$. We can confirm that the body gray-levels of the converted helicopter to SWIR and MWIR get darker, compared to LWIR model.

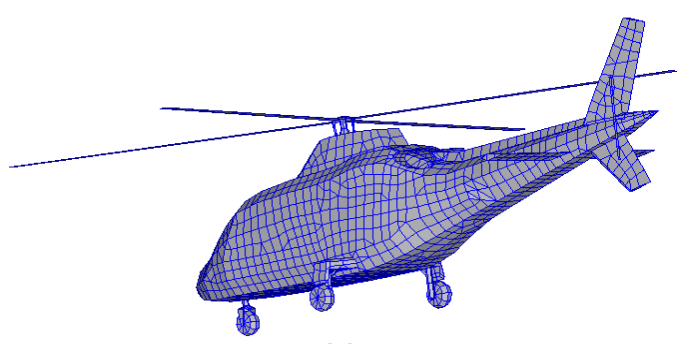

(a)

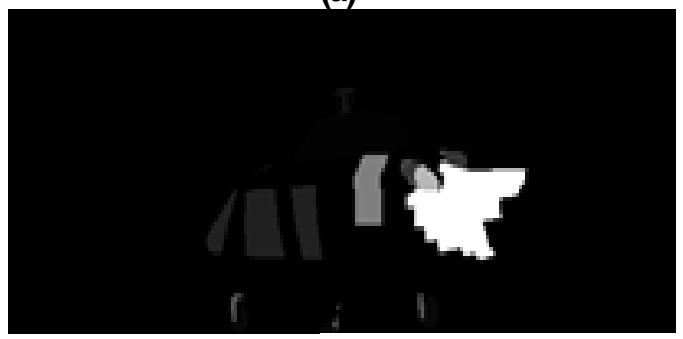

(c)

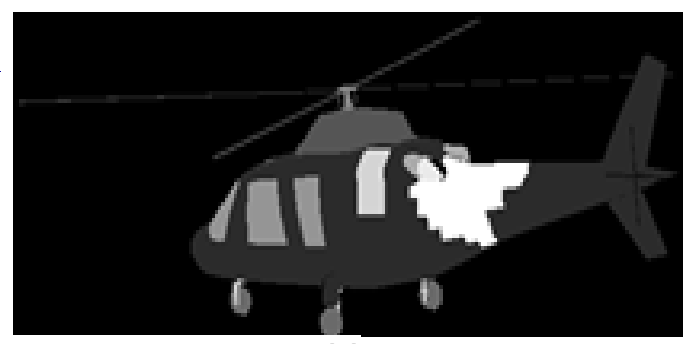

(b)

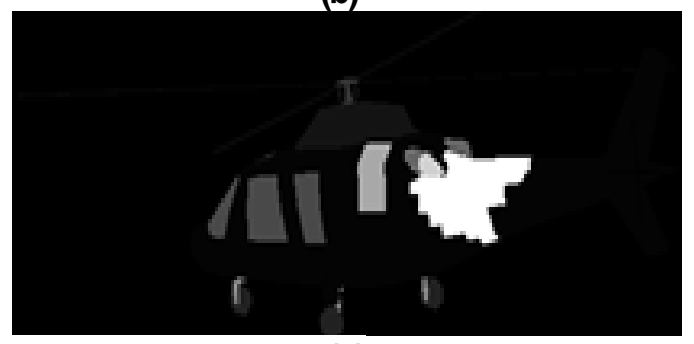

(d)

Figure 7. Result for wavelength-band transform: (a) 3D CAD model of Agusta helicopter, (b) original image of LWIR, converted images to (c) SWIR and (d) MWIR for modeled Agusta helicopter. 
Table 1. Result for wavelength-band transform for IR background image

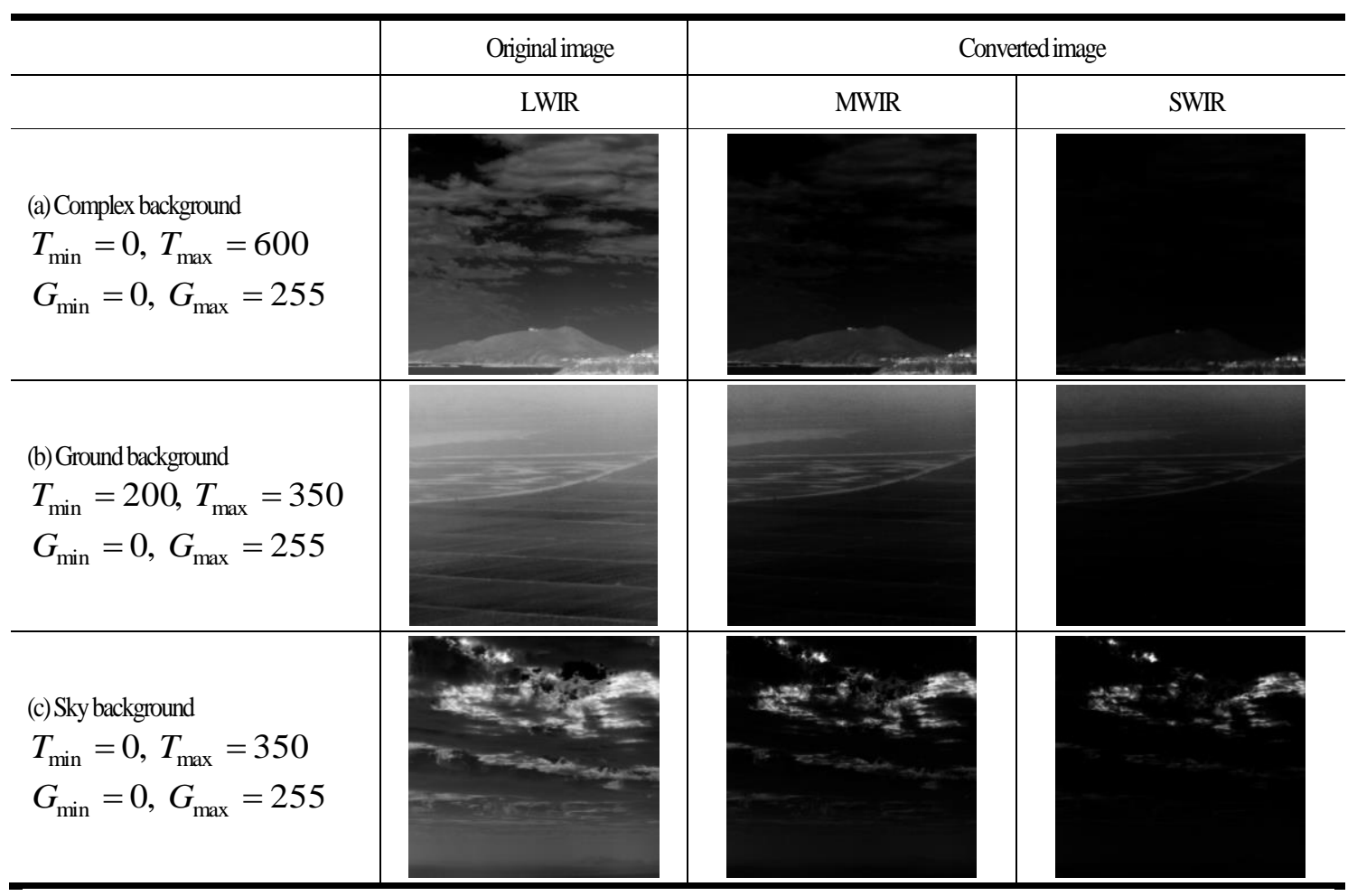

Also, original LWIR image (the first column) on Table 1 was captured by a thermal LWIR camera and the second and third column images represent the converted images to MWIR and SWIR. The image of Table 1(a) shows a complex IR background including sky and ground. And the image of Table 1(b) shows a ground IR background image with small temperature range. The image of Table 1(c) shows a sky IR background with high contrast. We can know that all the original LWIR images were transformed to IR images of MWIR and SWIR band.

Table 2. Temperature and emissivity of modeled helicopter

\begin{tabular}{ccc|ccc}
\hline Region & Title & Temperature $\left[{ }^{\circ} \mathrm{C}\right]$ & Region & Title & Temperature $\left[{ }^{\circ} \mathrm{C}\right]$ \\
\hline 1 & Tail rotor & 150 & 7 & Windows & 50 \\
2 & Fuselage & 60 & 8 & Exhaust port & 300 \\
3 & Rotorblade & 150 & 9 & Landing gear frame & 70 \\
4 & Rotor base & 200 & 10 & Engine cowling & 150 \\
5 & Tire & 50 & 11 & Engine inside & 600 \\
6 & Rotor shaft & 100 & 12 & Plume & 300 \\
\hline
\end{tabular}




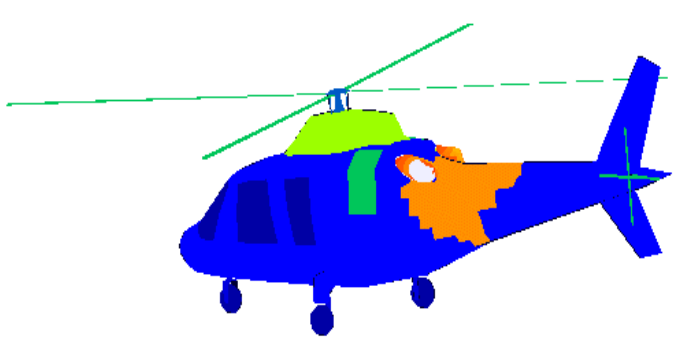

(a)

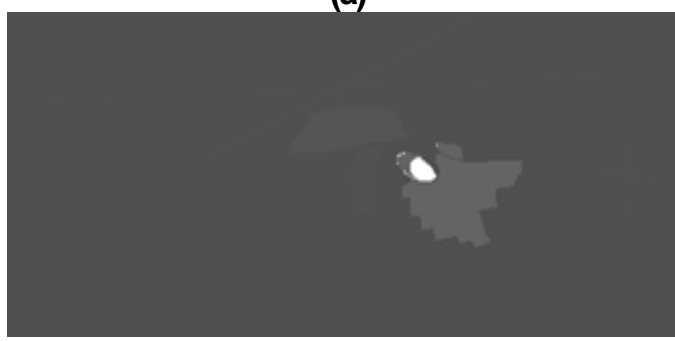

(c)

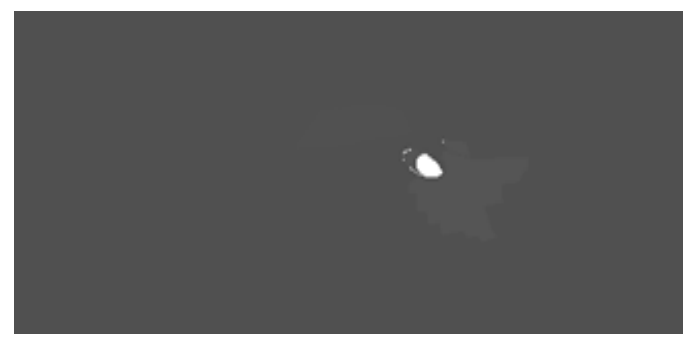

(b)

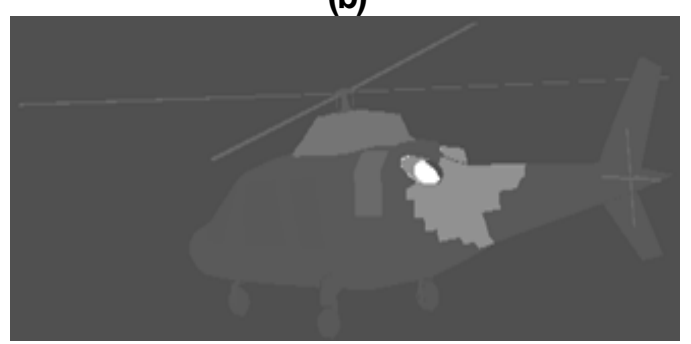

(d)

Figure 8. Temperature range image: (a) pseudo color image of estimated temperature, converted images to (b) SWIR, (c) MWIR, and (d) LWIR for modeled Agusta helicopter

In Figure 7(a), the Agusta helicopter was modeled by just temperature range and its corresponding gray-levels. As shown in Figure 8(a), in order to consider the temperature distribution the actual structure materials of the helicopter as mentioned in Table 2, the helicopter was modeled based on temperatures corresponding to these 12 structure materials. Figure $8(\mathrm{~b}) \sim(\mathrm{d})$ show respective LWIR, MWIR and SWIR images of original Agusta helicopter of Figure 8(a), transformed by Equations (7) (10).

\section{Conclusion}

This study suggested a wavelength-bandwidth transformation method of a real IR background and modeled IR target image. And surface temperature is obtained by calculating the radiance values for each part of the Agusta helicopter image. We confirmed that one image can be transformed to the other wavelength-band image, using actual IR background and modeled target image. The proposed method is expected to be applied in IR guided missile explorers and DIRCM system simulations, as it can actively cope with IR images at various forms.

\section{Acknowledgements}

This work was supported by a basic research project of ADD (Agency for defense development) in Korea

\section{References}

[1] R. G. Driggers, P. Cox and T. Edwards, "Introduction to infrared and electro-optical systems", Artech House, (1998).

[2] R. A. Jacobs, "Thermal Infrared Characterization of Ground Targets and Backgrounds", SPIE Optical Engineering Press, (1996). 
[3] D. H. Pollock, "Countermeasure systems”, The Infrared \& Electro-Optical Systems Handbook, vol. 7, SPIE, Optical Engineering Press, (1993).

[4] A. R. Jha, "Infrared Technology: Applications to electro-optics, photonic devices, and sensors", John-Wiley \&Sons, (2000).

[5] W. Yu, Q. Peng, H. Tu and Z. Wang, "An infrared image synthesis model based infrared physics and heat transfer”, International Journal of Infrared and Millimeter Waves, vol. 19, no. 12, (1998), pp. 1661-1669.

[6] C. X. Pan, J. Z. Zhang and Y. Shan, "Modeling and analysis of helicopter thermal and infrared radiation", Chinese Journal of Aeronautics, vol. 24, (2011) March, pp. 558-567.

[7] J. Lu and Q. Wang, "Aircraft-skin infrared radiation characteristics modeling and analysis", Chinese Journal of Aeronautics, vol. 22, no. 5, (2009), pp. 493-497.

[8] R. Dulski, T. Sosnowski and H. Polakowski, "A method for modelling IR image of sky and clouds", Infrared Physics \& Technology, vol. 54, (2011), pp. 53-60.

[9] H. K. Kim, S. H. Han, G. P. Hong and J. S. Choi, "Simulation of reticle using the generated thermal images", Proceedings of IEEE Asia Pacific Conf. on Circuits and Systems, (1996) November, pp. 18-21.

[10] L. J. Cox, M. A. Batten, S. R. Carpenter and P. A. B. Saddleton, "Modelling counter-measures to imaging infrared seekers", Proceedings of SPIE, vol. 5615, (2004), pp. 112-119.

[11] C. J. Wllers and M. S. Wheeler, "The validation of models in an imaging infrared simulation", Microwave and Optoelectronics Conference, (2007), pp. 250-254.

[12] C. J. Wllers and J. S. H. van den Bergh, "Optronics sensor development using an imaging simulation system", Electronics, Communications and Photonics Conference (SIECPC), (2011), pp. 1-6.

[13] M. Petersson, "Real-time DIRCM system modelling", Proceedings of SPIE, vol. 5615, (2004), pp. 149-160.

[14] S. H. Ahn and Y. C. Kim, "A wavelength-band translation method of infrared image", The Journal of Korean Institute of Information Technology, vol. 10, no. 7, (2012) (in Korean), pp. 87-96.

\section{Authors}

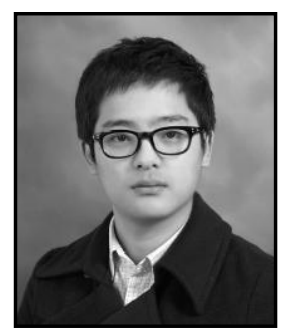

\section{Bong-Seob Kim}

$\mathrm{He}$ received the B.S. degree in Electric Engineering form Inje University, Korea, in 2012. He is currently pursuing the M.S. degree in School of Electronics Engineering from Kyungpook National University.

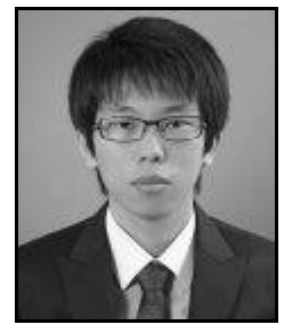

\section{Hyuk-Ju Kwon}

He received the B.S. and M.S. degree in School of Electronics Engineering form Kyungpook University, Korea, in 2010 and 2012. $\mathrm{He}$ is currently pursuing the Ph.D. degree from Kyungpook National University

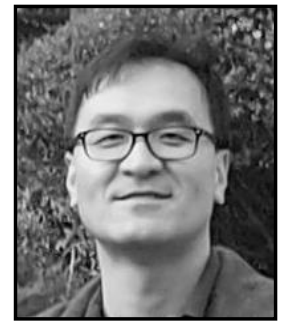

\section{Tae-Wuk Bae}

He received M.S., and Ph. D. degree in Electrical Engineering, Kyungpook National University, Korea, in 2006 and 2010, and then performed image-processing research as a postdoctoral researcher at KAIST, Korea in 2011 and Stanford University, USA in 2012. His research interests are image processing, pattern recognition, and infrared Search and Tracking. 


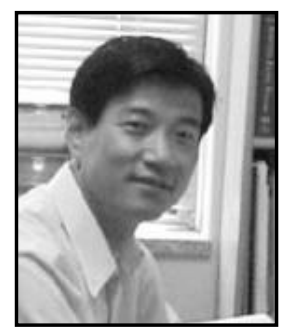

\section{Sang-Ho Ahn}

He received the B.S., M.S., and Ph. D. degree in Electrical Engineering from Kyungpook National University, Korea, in 1986, 1988, and 1992, and he joined the Electric Engineering at Inje University, Gimhae, Korea. His research interests are video signal processing, infrared image processing, and infrared countermeasures.

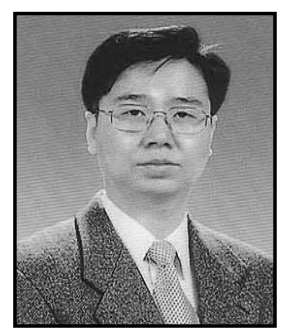

\section{Young-Choon Kim}

He received the B.S., M.S., and Ph. D. degree in Electrical Engineering from Kyungpook National University, Korea, in 1991, 1993, and 1997, and he joined the School of Dept. of Information and Communication Security at Youngdong University, Youngdong, Korea. His research interests are video signal processing, infrared image processing, and infrared countermeasures.

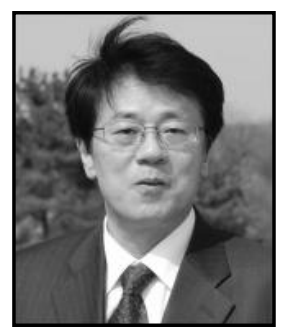

\section{Kyu-Ik Sohng}

He received his Ph.D. from Tohoku University, Sendai, Japan, in 1990. From March 1977 to February 1982, Dr. Sohng worked for the Agency for Defense Development, Daejeon, Korea, then in March 1982, he joined the School of Electronics Engineering at Kyungpook National University, Daegu, Korea. His current research interests include audio and video signal processing, color reproduction engineering, digital television, display \& health, and automotive electronics engineering. 
International Journal of Control and Automation Vol.6, No.6 (2013) 